\title{
Application of Integrated Human Error Management in Human Factors Engineering Process to Nuclear Power Plant Design
}

\author{
Kenji Mashio \\ ICT Solution Headquarters, \\ Mitsubishi Heavy Industries, Ltd., Kobe, Hyogo, Japan. \\ Corresponding author: kenji.mashio.7c@nu.mhi.com \\ Kodo Ito \\ Department of Engineering, \\ Tottori University, Tottori, Japan. \\ E-mail: itokodo@tottori-u.ac.jp
}

(Received on January 27, 2021; Accepted on June 23, 2021)

\begin{abstract}
Integrated process of human error management in human factors engineering (HFE) process provides a systematic direction for the design countermeasures development to prevent potential human errors. The process analyzes performance influence factors (PIFs) for crew failure modes (CFMs) and human failure events (HFEvs) in human reliability analysis (HRA). This paper provides applications of the process to the event evaluation for nuclear power plant design, especially PWR. In this application, the HRA/HFE integrated process had specified further detail for PIF attributes which had not been obtained in HRA, and showed further investigations to treat how operators induced their human errors through their cognitive task process in their work environment. This application showed effectiveness of the process in order to provide design countermeasures for preventing potential human errors occurrence based on the extensive PIFs and their error forcing context in HRA.
\end{abstract}

Keywords- Human factors engineering, Human reliability analysis, Human error management.

\section{Introduction}

Human Factors Engineering (HFE) process is applied to control room design. Figure 1 shows a typical HFE process applied in control room design (U.S.NRC, 2012).

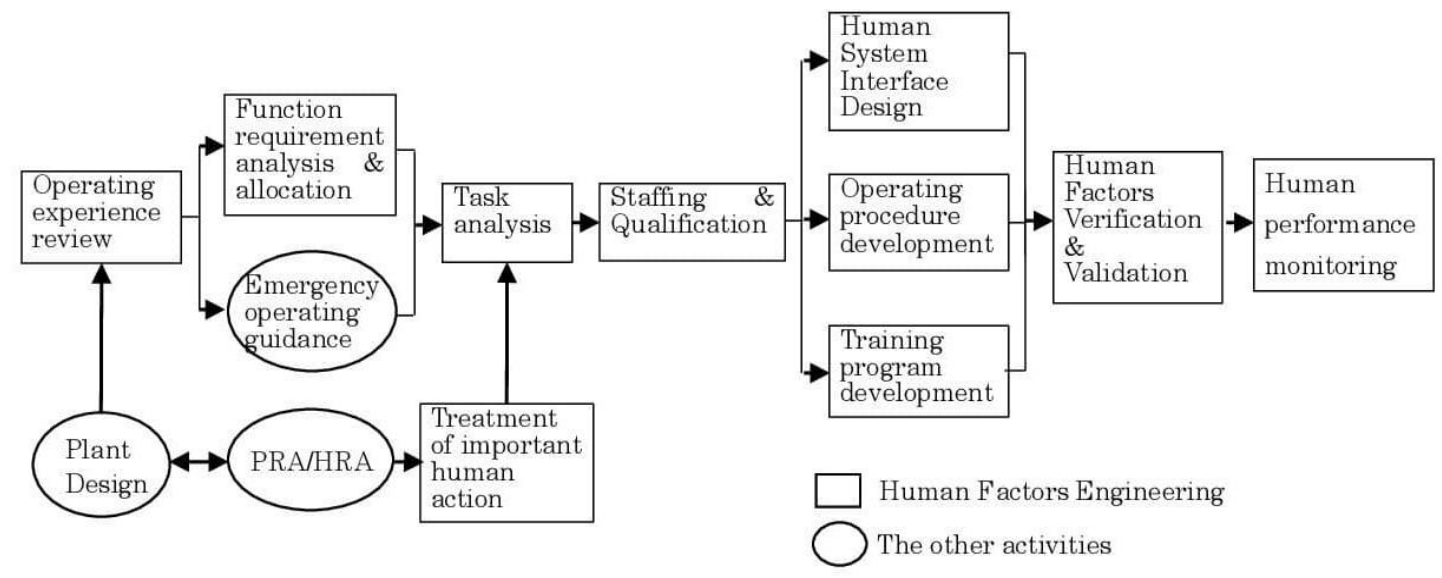

Figure 1. HFE process (U.S.NRC, 2012). 
The current practice is to extract important human actions (RIHAs) in the "treatment of important human action" process by applying to risk-importance measures (U.S.NRC, 2007). Riskimportance measures are metrics to measure contributions of risk significance value (i.e., Core damage frequencies (CDFs)) due to particular event (i.e. functions/components failed). Two of different but complementary risk-importance measure are applied; Risk Achievement Worth (RAW) and Fussell-Veselly (FV). The RAW measures the amount that the total risk increases if particular event occurs, and is defined as RAW $=(\mathrm{CDFs}$ with $\mathrm{A}=1) /(\mathrm{CDFs})$, where CDFs with $\mathrm{A}$ $=1$ means probability of event $\mathrm{A}$ (failure event) occurrence is set to 1 . The FV measures the overall percent contribution of containing particular event against the total risk, and is defined as FV = (CDFs with $\mathrm{A}) /(\mathrm{CDFs})$, where $\mathrm{CDF}$ s with A means all cut set of risks which $\mathrm{A}$ are involved. RIHAs are extracted if either of RAW and FV is higher than particular thresholds. However, it is difficult to figure out what operator crew's failure modes (CFMs) or their performance influence factors (PIFs) are involved in particular events. After identifying IHAs, task analysis in HFE process individually performs for IHAs in order to identify PIFs factors and design requirement, such as plant parameters/controllers necessary for task executions.

Human reliability analysis (HRA) aims to calculate human error probabilities (HEPs) tied with probabilistic risk analysis (PRA). For this purpose, HRA consists of two parts; qualitative analysis and quantitative analysis. The qualitative analysis identifies human failure events (HFEvs) in PRA events, then analyzes each HFE to find out operator crew's failure modes (CFMs) and their performance influence factors (PIFs) involved in each HFE. Then the quantitative analysis calculates HEPs by cumulating each HEP in CFMs/PIFs involved in each HFE. HRAs have been enhanced to ensure accuracy and consistency of HEP calculations. The latest HRA methods (U.S.NRC, 2020) introduced a macro-cognitive model which provides a set of cognitive CFM types and their PIFs and detail work scheme/process that allow practitioners to identify comprehensive cognitive errors with their PIFs. Figure 2 shows an example of HRA process (U.S.NRC, 2020).

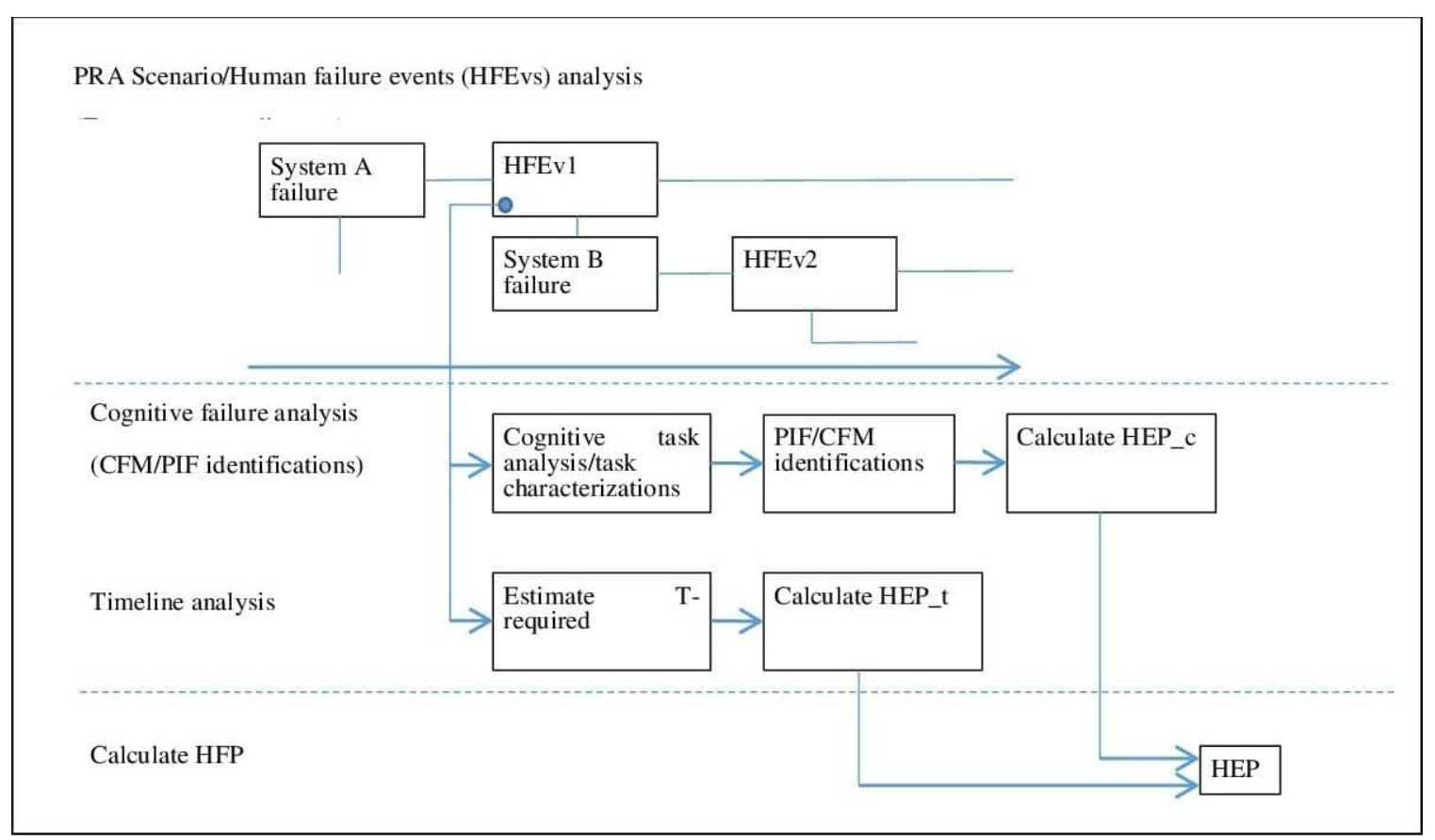

Figure 2. HRA process (U.S.NRC, 2020). 
It is effective if HRA assumptions, particulary qualitative analysis results (i.e., CFMs/PIFs) are directly communicated with HFE process (i.e., task analysis) not just by screening with risk importance thresholds, then CFMs/PIFs which are currently used for HEPs calculations, are used for human system interface (HSI) development and procedure development as a design requirement in HFE process in order to minimize potential human error occurrences. The previous study (Boring et al., 2008) provided a possibility and framework to integrate HFE and HRA process.

In order to realize the integration, the current interface between HRA/PRA and HFE process must be modified and demonstrated. This paper provides a study how to integrate HRA and HFE process by employing the latest HRA qualitative process (U.S.NRC, 2020), then demonstrate the integration process with the sample event showing the difference in the current HRA quantitative analysis results.

\section{Integration of HRA in HFE Process}

The integration process with HRA quantitative analysis employed is described in Figure 3.

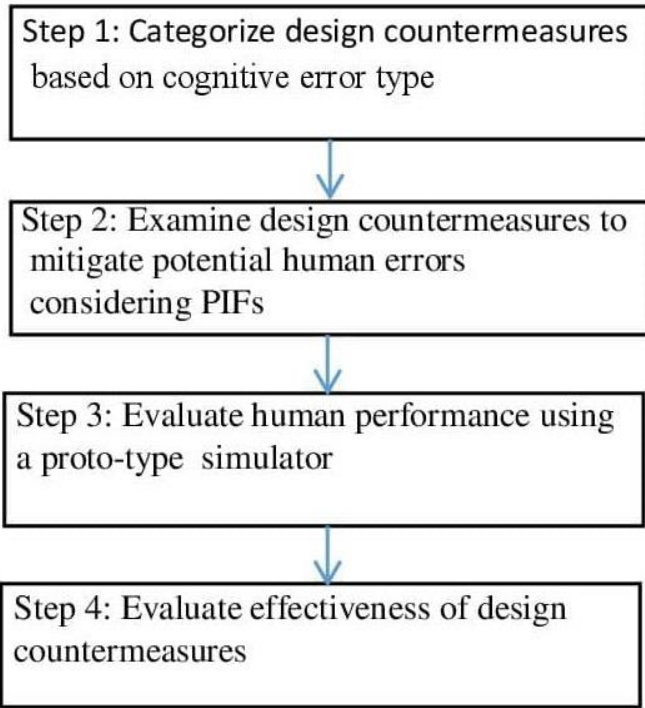

Figure 3. HRA/HFE integrated process.

Each step is performed with following activities:

Step 1: Categorize design countermeasures based on cognitive error types

Overall design countermeasures are examined based on cognitive error types and PIFs attributes. Design countermeasures are taken into account from comprehensive aspects; HSI designs (including workplace and work environment design), operating procedures and operator training based on PIF types. Those design countermeasures are still generic means and may already have been addressed by existing HFE design guide.

Step 2: Examine design countermeasures to mitigate potential human errors considering PIFs This step analyzes context descriptions and relations of task sequences and CFMs/PIFs. The step also analyzes task frequency or iteration during events which cause potential human error increase. 
From error management point of view, design countermeasures are examined by both of error preventions and mitigations as shown in Figure 4. Mitigations generally mean to minimize hazard expansions but from human factors aspects, error of commissions (EOCs) becomes a major factor to progress/expand error affections after initial human error occurrence. Therefore, the mitigations of hazard, which are caused by human errors, focus on how to minimize EOCs during events.

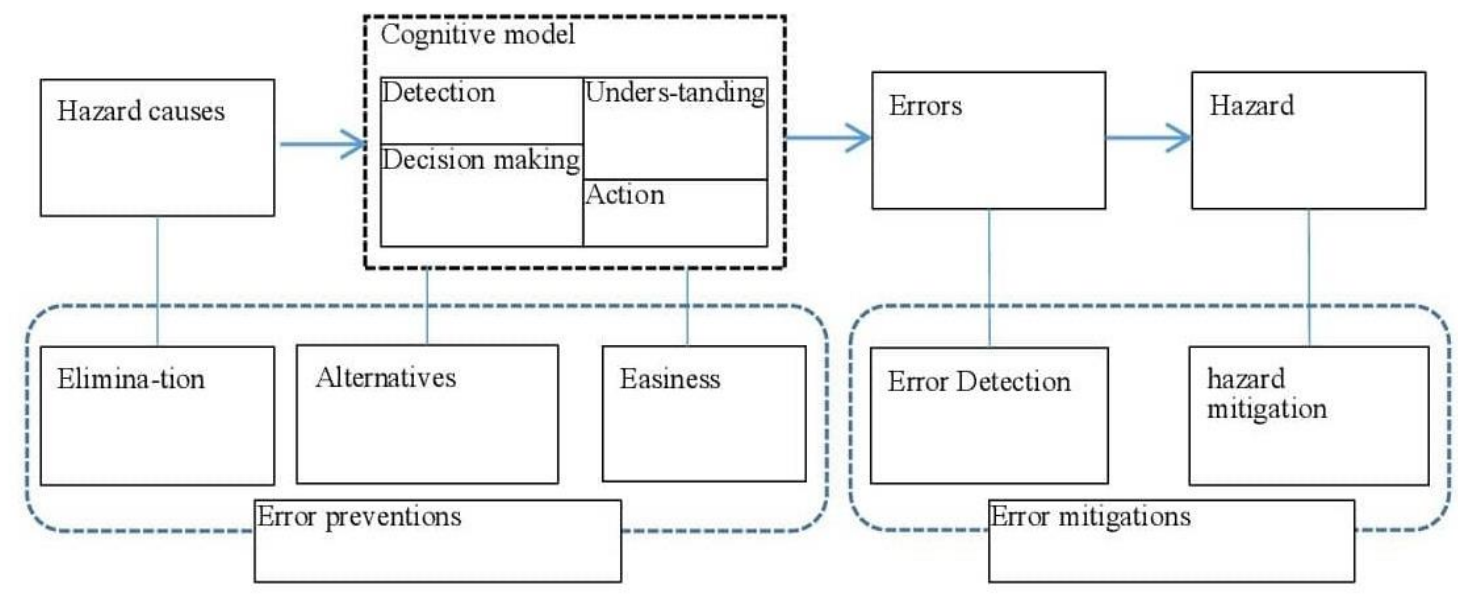

Figure 4. Error prevention process.

Followings are results from examinations of each error elimination and mitigation process;

(a). Error preventions

As long as nuclear power plants are operated, hazard causes are not eliminated. Alternatives can be made by appropriate auto/manual allocations based on task analysis. If particular tasks require human capabilities challenging, such as high workload, then automation is recommended. Easiness is to make tasks executions easier considering human cognitive process.

(b). Error mitigations

Error detection means include work management tools and methods in operating procedures/training programs, such as, check sheet on procedure steps, three way commutations between operators and supervisors. In case of EOC corrections, third party checks, such as independent plant event diagnosis by technical support staffs and/or computer aids, are effective.

Hazard mitigations are predetermined by plant system designs, then mitigation means are provided and installed in plant site and operating procedure address how those mitigation means are used in accordance with plant conditions. In NPPs, plant event diagnostics are credited by operators. Correction of EOC is a basis toward mitigations.

Step 3: Evaluate human performance using a proto-type simulator Design countermeasures which are examined in Step 1 and 2, are evaluated using a simulator.

Step 4: Evaluate effectiveness of design countermeasures

In order to identify design countermeasure effectiveness, comparison checks with design 
countermeasure implemented or not are performed with the same work environment, scenarios and staffs in order to eliminate the other influence factors or biases. Digital HSIs are relatively easier to switch either of design options in a simulator.

\section{Process Evaluation Using a Sample of Plant Event Integration of HRA in HFE Process}

Following sub-sections shows examination result to execute the process. Steam generator tube rapture (SGTR) event was selected to compare HRA/HEF integration results with the original results discussed in the HRA (U.S.NRC, 2020).

\subsection{Step 1}

The sample of examination result is shown in Table 1. The design resolution is allocated to each PIF type but does not specify each specific number of PIF within the same type of PIF. Therefore, design counter measures are allocated in each representative PIF type. Each bold marked line shows major design counter measures. Parentheses show subsidiary means.

Table 1. PIFs/Design countermeasures comparison matrix (U.S.NRC, 2020).

\begin{tabular}{|c|c|c|c|c|c|c|c|c|}
\hline & \multicolumn{5}{|c|}{ Macro-cognitive function } & \multicolumn{3}{|c|}{ Design Countermeasures } \\
\hline PIF Attribute & D & $\mathrm{U}$ & $\mathrm{DM}$ & A & $\mathrm{T}$ & HSI & Procedures & Training \\
\hline \multicolumn{9}{|l|}{ Base PIF } \\
\hline $\begin{array}{l}\text { Scenario } \\
\text { Familiarity }\end{array}$ & & & & & & $\begin{array}{l}\text { (Confirm HSI } \\
\text { to consider to } \\
\text { execute the } \\
\text { scenarios) }\end{array}$ & $\begin{array}{l}\text { (Confirm } \\
\text { Procedures to } \\
\text { cover the } \\
\text { scenarios) }\end{array}$ & $\begin{array}{l}\text { Enhance } \\
\text { frequency } \\
\text { training }\end{array}$ \\
\hline SF0: No impact & $1 \mathrm{E}-4$ & $1 \mathrm{E}-3$ & $1 \mathrm{E}-3$ & $1 \mathrm{E}-4$ & $1 \mathrm{E}-3$ & & & \\
\hline $\begin{array}{l}\text { SF1: Unpredictable } \\
\text { dynamics in known } \\
\text { scenarios }\end{array}$ & $6.6 \mathrm{E}-4$ & $6.6 \mathrm{E}-3$ & $6.6 \mathrm{E}-3$ & $6.6 \mathrm{E}-4$ & N/A & & & \\
\hline $\begin{array}{l}\text { SF2: Unfamiliar } \\
\text { elements in the } \\
\text { scenarios }\end{array}$ & $5 \mathrm{E}-3$ & $5 \mathrm{E}-2$ & $5 \mathrm{E}-2$ & $5 \mathrm{E}-3$ & N/A & & & \\
\hline $\begin{array}{l}\text { SF3-1: Scenarios } \\
\text { trained on but } \\
\text { infrequently } \\
\text { performed }\end{array}$ & $1 \mathrm{E}-3$ & $1 \mathrm{E}-2$ & $1 \mathrm{E}-2$ & $1 \mathrm{E}-3$ & N/A & & & \\
\hline $\begin{array}{l}\text { SF-3-2: Scenario is } \\
\text { unfamiliar, rarely } \\
\text { performed }\end{array}$ & $1.2 \mathrm{E}-2$ & 1E-1 & $1 \mathrm{E}-1$ & $3.3 \mathrm{E}-2$ & N/A & & & $\begin{array}{c}\text { Enhance Coping } \\
\text { skill by training }\end{array}$ \\
\hline $\begin{array}{l}\text { SF-3-3: Extremely } \\
\text { rarely performed }\end{array}$ & $3.3 \mathrm{E}-2$ & $3 \mathrm{E}-1$ & $3 \mathrm{E}-1$ & $3.5 \mathrm{E}-1$ & N/A & & & \\
\hline $\begin{array}{l}\text { SF-4: Bias or } \\
\text { preference for } \\
\text { wrong strategies } \\
\text { exists, mismatched } \\
\text { mental models }\end{array}$ & N/A & $2.6 \mathrm{E}-2$ & $2.6 \mathrm{E}-2$ & N/A & N/A & & & \\
\hline
\end{tabular}

Note) D: Detection, U:Understanding, DM: Decision Making, A: Action Execution, T: Team Coordination. 
Followings are obtained in Step 1 process:

(i) The same design countermeasure is attributed to each type of PIF category (i.e., not depending on human error rate scale)

(ii) Existing ergonomics design guides already covered design countermeasures which are allocated against PIFs based on generic PIF reviews.

(iii) Specific ergonomics design guides are difficult to be assigned to each PIF type because each PIF is still generic description.

(iv) Prioritization based on PIF human error rate scale is effective if it is combined with scenarios, which is discussed in Step 2.

\subsection{Step 2}

Step 2 is divided by following sub-steps;

Sub-step 2-1: Identification of initial PIFs and CFMs/HFEvs extracted from existing HRA results (U.S.NRC, 2020) executed by following process:

(i) Scenario analysis, Perform narrative description development and timeline analysis, then identify HFEvs and initial PIFs.

(ii) HFEv analysis, Perform operating sequence analysis, then identify critical tasks.

(iii) Perform cognitive tasks analysis, then identify CFMs. Perform task characterizations and precise initial PIFs.

(iv) Calculate human error probabilities (HEPs) for each critical task by accumulating human error rates of CFMs/PIFs involved in critical tasks.

There are many information split in HRA so that the first step is to review HRA results, extract necessary information, including initial PIFs and CFMs/HFEvs which are necessary for subsequent analysis steps.

Sub-step 2-2: PIF type in HRA cover broad areas, including HSI, procedures and training. However, HRA focuses on identifying based PIFs or significant PIFs, which largely influence CFMs/HFEvs. Therefore, an additional analysis reviews HRA's identification PIFs and add PIFs considering work system's influence factors, such as HSI, operating procedure, and training which are subsidiary influence factors and may vary due to particular type of control rooms/plants.

Sub-step 2-3: Operation frequency analysis. HRA performs the analysis based on operating procedure. Human error rate estimation for each PIF is one time action basis. This step reflects operator's behaviors and causal operator's tasks based on plant response during scenarios, which are not always identified from operating procedure based analysis. That is, especially, frequencies of operations for the same actions, which may not be takin into account in HRA and largely, impact on human performance. Therefore, operation frequencies for the same actions are counted and taken into account as additional weight factors.

Sub-step 2-4: Examine design countermeasures from error preventions/mitigations. Error preventions provides pre-design design solutions as error proof and cover HSI design (including work environment), operating procedures, staffing (including organizations and training). Error mitigations focus on providing design solution from how operator acknowledge abnormal situation, then minimize EOC during event. Design solution for error mitigation includes operation support functions using computer aids or communications provided by operation support personnel. 
In addition, important/critical operator's actions from deterministic and risk analysis will be determined if plant events are determined due to plant situation progress. Therefore, those operator's important/critical actions are displayed on operating support personnel display screen at real time basis, they may support operators which actions are prioritized for plant responses.

The summary of Step 2 examination is as follows:

\section{Results of Sub-step 2-1:}

Following are the summary extracting necessary information from HRA results for SGTR (U.S.NRC, 2020, Appendix C);

(i). Following tasks were identified as HFEvs;

HFEv-T1: Fail to isolate the ruptured SG.

Following PIFs were identified based on context analysis; Task complexity, Procedures, Multitasking/ interruption/distraction, Stress and time pressure.

(ii). HFEvs analysis

Task diagram was developed and two key tasks were identified as shown in Figure 5 (U.S.NRC, 2020). The first task was assigned to automation, then the second was determined as a critical task;

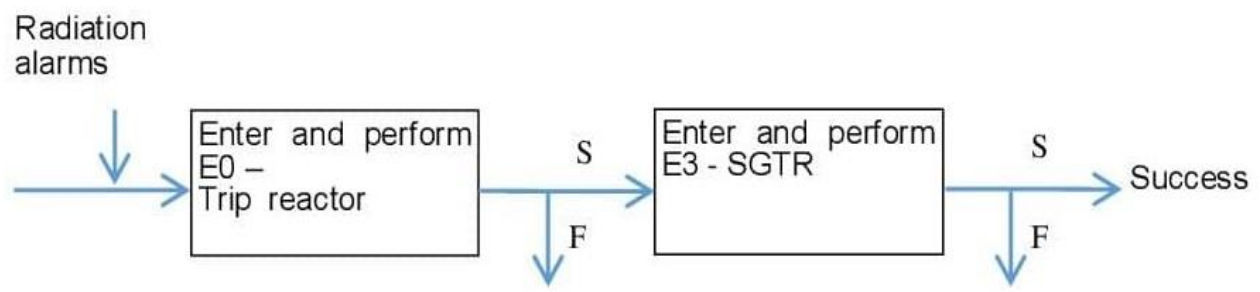

Figure 5. Task diagram in HRA (U.S.NRC, 2020).

(iii). Cognitive task analysis

Cognitive tasks analysis in HFEv-T1 was performed, then following three CFMs were identified:

T1-CFM1: Failure of Detection

T1-CFM2: Failure of Understanding

T1-CFM4: Failure of Execution

Following task characteristics were identified in HFEv1-T1 (Omitting PIFs assigned to "Noimpact").

Special requirements - The task needs to be performed before reaching the SGPORV setpoints.

Cue - The cue to start the task is the secondary radiation alarm.

Personnel - Adequate well-trained crew.

Procedure -EOP-0 and EOP-3 have been implemented in simulator training. The procedures have been optimized based on training feedback.

Following initial PIFs were identified. It is noted that all PIF codes are extracted from the HRA (U.S.NRC, 2020, Appendix B). The description of each PIF code are added after each PIF code. 
For example, "C0-No impact" means there is a base task complexity without additional impact;

\section{T1-CFM1}

Task complexity: $\mathrm{C} 0$ - No impact.

Procedures: P0 - No impact.

Multitasking: MT0 - no need for multitasking.

Mental Fatigue, stress and time pressure: FS0 - No impact.

\section{T1-CFM2}

Task complexity: C30 - No impact.

Procedures: PG0 - No impact.

Multitasking: MT0 - no need for multitasking.

Mental Fatigue, stress and time pressure: FS0 - No impact.

\section{T1-CFM4}

Task complexity: C42 - EOP-3 has multiple proceduralized steps and some steps need control manipulation.

Procedures: P0 - No impact.

Multitasking: MT0 - no need for multitasking.

Mental Fatigue, stress and time pressure: FS0 - No impact.

Where C0, P0, MT0, etc., are indicated as PIF types and influence attribute (e.g., C0 means "Task complexity - No impact").

(iv). HEP estimation

Table 2 shows summary of HFEv1, critical task involved in HFEv1, applicable CFMs and PIFs, then calculates HEP based on accumulations of applicable PIF's human error rates.

Table 2. Summary of CFMs/PIFs in HFEv1.

\begin{tabular}{|c|c|c|}
\hline Critical Task & Applicable CFMs & Applicable PIF attribute \\
\hline \multirow{3}{*}{$\begin{array}{l}\text { HFEv1-T1: Enter and perform } \\
\text { EOP-3 }\end{array}$} & T1-CFM1: failure of Detection & No impact \\
\hline & $\begin{array}{lll}\text { T1-CFM2: } & \text { failure } & \text { of } \\
\text { Understanding } & & \\
\end{array}$ & No impact \\
\hline & T1-CFM4: failure of Execution & $\begin{array}{l}\text { Task complexity C42 - Multiple } \\
\text { simple proceduralized steps with control manipulations }\end{array}$ \\
\hline
\end{tabular}

\section{Results of Sub-step 2-2:}

Following are a summary of additional analysis from HFE/HRA perspectives;

With the task analysis results, which have comprehensively been performed in HFE process, an extensive analysis was performed to identify following HFEvs and CFMs/PIFs;

(a). Check reactor trip \& turbine trip

CFM1: PIFs-C1, HSI 2, 3, 5, EVN7, FS6, SF0, Inf0;

Note) EVN7 (Loud or burst noise) and FS6 (Sudden increase in workload from a long period of low to high) have been selected because a lot of numbers of alarms are initiated at reactor trip (EVN7), and sudden increase of workload is expected at reactor trip from a long period of low workload at a normal operation. HSI 2, 3, and 5 were selected depended on HSI design qualities 
(2: No sign or indication of technical difference from adjacent sources (meters, indicators), 3 : Related information for a task is spatially distributed, not organized, or cannot be accessed at the same time, 5: Poor salience of the target (indicators, alarms, alerts) out of the crowded background). HSI6 (Inconsistent formats, units, symbols, or tables etc.,) was not selected because Step 1 (generic ergonomics design review and applications) eliminate this influence factor. HSI 5 was selected for HEP calculation for safety margin consideration.

CFM4: PIFs- C31, HSI2,3,5, EVN7, FS6, SF0, Inf0

C31 (Straightforward Procedure execution with many steps) was selected because a precise operating procedure for reactor trip response provides straightforward steps but many steps including component status checks and verifications. EVN7, FS6, and HSI3,4,5 selections were the same reason as those of CFM1.

(b.) Check Safety Injection (SI) status

CFM1: PIFs- C1, HSI2,3,5, EVN7, FS6, SF0, Inf0

CFM4: PIFs- C31, HSI2,3,5, EVN7, FS6, SF0, Inf0

Note) Selection of PIFs is the same situation as item a.

(c.) Check Emergency Feed water (EFW) valve alignment

CFM1: PSF- C31, HSI2,3,5, FS6, SF0, Inf0

Note) EVN7 was not selected because many additional alarms are not expected to initiate after this step. FS6 was selected because this step is a series of tasks in item a. and b. The other PIFs selections are the same reasons as a. and $b$.

(d). Control SG level

CFM1: PSF- C0, HSI2,3,5, FS8, MT1, SF0, Inf0

CFM4: PSF- C31, HSI2,3,5, FS8, MT1, SF0, Inf0

Note) SF8 (Emotional stress (e.g. anxiety, frustration)) was selected because emotional stress is possibly emerging whereas sudden increase of workload was calmed down after a series of tasks in a., b. and c.

MT1 (Distraction by other ongoing activities that demand attention) was selected because operators are required to pay much attentions to manual control of SG level in parallel with the other step executions during SGTR.

(e). Diagnose $\mathrm{SG}$ tube rapture

CFM1: PSF- C3, HSI2,3,5, FS8, SF0, Inf0

C3 (Detection demands for high attention) was selected because this task (detection) requires operators to carefully monitor the secondary radiation alarm initiation which is a key parameter to diagnose SGTR. This task and subsequent tasks correspond to HFEv1-T1 as identified in HRA.

CFM4: PSF- C31, HSI2,3,5, FS2, FS8, SF0, Inf0

(f). Isolate flow from ruptured $\mathrm{SG}$

CFM4: PIF- C31, HSI2,3,5, FS2, FS8, SF0, Inf0

(g). Cool down RCS temperature \& pressure

CFM1: PIF- C0, HSI2,3,5, FS8, MT1, SF0, Inf0

CFM4: PIF- C31, HSI2,3,5, FS8, MT1, SF0, Inf0

MT1 was selected because operators perform RCS cooldown by monitoring subcooling margin and 
executing the other steps in parallel.

Table 3 shows the summary of HFEvs and associated information from HRA/HFE extensive analysis as discussed in this sub-step.

By comparing with results from the original HRA based results as shown in Table 2, extensive critical tasks are identified with additional PIFs attribute. It is noted that all critical tasks are categorized in the same HFEv (i.e., HFEv1) in this study since all critical tasks are related to HFEv1.

Table 3. Summary of CFMs/PIFs in critical tasks.

\begin{tabular}{|c|c|c|}
\hline Critical Tasks & Applicable CFMs & Applicable PIF attribute \\
\hline \multirow{2}{*}{$\begin{array}{l}\text { HFEv1-T1: Check reactor trip } \& \\
\text { turbine trip }\end{array}$} & T1-CFM1: failure of Detection & $\mathrm{C} 1, \mathrm{HSI} 2,3,5, \mathrm{EVN} 7, \mathrm{FS} 6, \mathrm{SF} 0$, Inf0 \\
\hline & T1-CFM4: failure of Execution & C31, HSI2,3,5, EVN7, FS6, SF0, Inf0 \\
\hline \multirow{2}{*}{$\begin{array}{l}\text { HFEv1-T2: Check Safety Injection } \\
\text { (SI) status }\end{array}$} & T2-CFM1: failure of Detection & C1, HSI2,3,5, EVN7, FS6, SF0, Inf0 \\
\hline & T2-CFM4: failure of Execution & C31, HSI2,3,5, EVN7, FS6, SF0, Inf0 \\
\hline \multirow{2}{*}{$\begin{array}{l}\text { HFEv1-T3: Check Emergency } \\
\text { Feedwater (EFW) valve alignment }\end{array}$} & T3-CFM1: failure of Detection & C31, HSI2,3,5, FS6, SF0, Inf0 \\
\hline & T3-CFM4: failure of Execution & N/A \\
\hline \multirow{2}{*}{ HFEv1-T4: Control SG level } & T4-CFM1: failure of Detection & C0, HSI2,3,5, FS8, MT1, SF0, Inf0 \\
\hline & T4-CFM4: failure of Execution & C31, HSI2,3,5, FS8, MT1, SF0, Inf0 \\
\hline \multirow{2}{*}{ HFEv1-T5: Diagnose SG tube rapture } & T5-CFM1: failure of Detection & C3, HSI2,3,5, FS8, SF0, Inf0 \\
\hline & T5-CFM4: failure of Execution & C31, HSI2,3,5, FS2, FS8, SF0, Inf0 \\
\hline \multirow{2}{*}{$\begin{array}{l}\text { HFEv1-T6: Isolate flow from ruptured } \\
\text { SG }\end{array}$} & T6-CFM1: failure of Detection & N/A \\
\hline & T6-CFM4: failure of Execution & C31, HSI2,3,5, FS2, FS8, SF0, Inf0 \\
\hline \multirow{2}{*}{$\begin{array}{l}\text { HFEv1-T7: Cool down } \\
\text { temperature \& pressure }\end{array}$} & T6-CFM1: failure of Detection & C0, HSI2,3,5, FS8, MT1, SF0, Inf0 \\
\hline & T6-CFM4: failure of Execution & C31, HSI2,3,5, FS8, MT1, SF0, Inf0 \\
\hline
\end{tabular}

\section{Results of Sub-step 2-3:}

Item $\mathrm{d}$ and $\mathrm{g}$ tasks are frequent tasks which require operator to keep attention to plant process and initiate tasks if conditions are met during scenarios. Therefore, numbers of frequencies are set to 10 for item $\mathrm{d}$. and g. whereas the other tasks are set to 1 .

\section{Results of Step 2-4:}

A sample of results (for item a and e) are summarized in Figure 6. For example, as a design countermeasure, task support display (guidance display) is provided to operators to collect associated plant parameters and components status which are required to be checked operators. This design countermeasure can eliminate HSI2 and 3 influence factors. Also, demarcation (i.e. grouped and placed in dedicated area) of reactor and turbine trip alarms from the other alarms can eliminate HSI5 influence factor. These design counterparts can also eliminate C3 factor (i.e., Monitor many parameters, many types or categories of information to be detected). Regarding error mitigation means, for example, plant computer checks plant parameters and components status with reactor trip status, then display checking results to operators. Also, guidance display which displays associated plant parameters and components and/or plant computer checking result are displayed to plant operation support personnel, then plant operation support personnel can support operators to promote recovery actions, including operator's EOC corrections. 
a. Check reactor trip \& turbine trip

Diagnose SG tube rapture
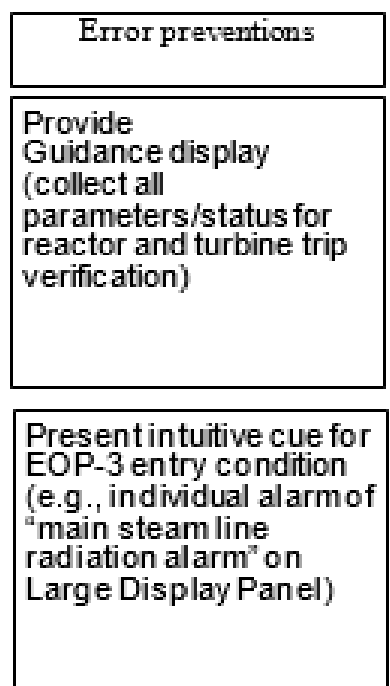

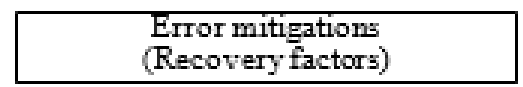

Present computer check results to operators (i.e., check whether all parameters/status meet reactor/turbinetrip criteria Present a list of critical tasks with task execution status and associated plant parameters to Operation support personnel.

\section{Present computer check results to operators. \\ Present a list of critical tasks with task execution status and associated plant parameters to Operation support personnel.}

Figure 6. Design countermeasures.

Another example is to display important/critical operator's actions (in this case, display "isolate ruptured SG within ten minutes") to plant operation support personnel so that they can support operators to direct goal to achieve safety operation (Figure 7).

Above countermeasures have already been incorporated in plant design based on performance review and/or design resolution examinations to dispose performance challenge using the integrated system validation and operating experience. However, those design resolutions are confirmed effective from human error preventions and mitigations aspect. Those design countermeasures are also identified as recovery factors for HRA, which could improve human error rate.

\begin{tabular}{|c|c|c|c|}
\hline \multicolumn{4}{|c|}{ Critical task list (SGTR) } \\
\hline Step\# & Critical tasks & $\begin{array}{l}\text { Associated plants/ } \\
\text { Component status }\end{array}$ & $\begin{array}{l}\text { Task goal } \\
\text { (criteria) }\end{array}$ \\
\hline $3-14$ & $\begin{array}{l}\text { Isolate flow from } \\
\text { reputed SG }\end{array}$ & $\begin{array}{l}\text { MSIV(VXXX) Open } \\
\text { MSRV(VXXX) Open }\end{array}$ & $\begin{array}{l}\text { Close within } 10 \text { minutes } \\
\text { Close }\end{array}$ \\
\hline $3-20$ & $\begin{array}{l}\text { Cool down RCS } \\
\text { Temperature } \\
\& \text { pressure }\end{array}$ & $\begin{array}{l}\text { RCS subcooling } \mathrm{XXX}^{\circ} \mathrm{C} \\
\text { Rate } \mathrm{Xx}{ }^{\circ} \mathrm{C} / \mathrm{hr}\end{array}$ & RCS subcooling \\
\hline
\end{tabular}

Figure 7. Critical task list display. 


\subsection{Step 3}

Evaluation of human performance and effectiveness introducing design countermeasures is performed by comparing results with design countermeasures vs without them. In general, workload (WL) and situation awareness (SA) are two major metrics to measure human performance especially for large and complicated human system interaction systems (Kirwan and Ainisworth, 1992).

Table 4 summarizes an example of WL and SA evaluation results applying a major SA and WL measurement methods (i.e., five points Likert scales and NASA TLX Hart and Staveland, 1988).

Table 4. Summary of design countermeasures.

\begin{tabular}{|c|c|c|c|c|}
\hline Critical tasks & $\begin{array}{l}\text { CFMs/ } \\
\text { PIFs }\end{array}$ & $\begin{array}{l}\text { SA } \\
\text { (5 pt. scale) }\end{array}$ & $\begin{array}{l}\text { Workload } \\
\text { (TLX-Overall/Major } \\
\text { weight) }\end{array}$ & Design Implemented \\
\hline $\begin{array}{l}\text { a. } \\
\text { Check reactor } \\
\text { trip \& turbine } \\
\text { trip }\end{array}$ & $\begin{array}{l}\text { CFM1:PIF- C1, } \\
\text { HSI2,3,5, } \\
\text { EVN7, FS6, } \\
\text { SF0, Inf0 } \\
\text { CFM4:PIF- } \\
\text { C31, HSI2,3,5, } \\
\text { EVN7, FS6, } \\
\text { SF0, Inf0 }\end{array}$ & $\begin{array}{l}\text { WO/ DS: } \\
3.1 \\
\text { W/ } \\
\text { DS: } \\
4.0\end{array}$ & $\begin{array}{l}\text { WO/ DS: } \\
68 \% \\
\text { W/ } \\
\text { DS: } \\
50 \%\end{array}$ & $\begin{array}{l}\text { Error previsions: Guidance display (collect all } \\
\text { parameters/ status for reactor and turbine trip } \\
\text { verification)(HSI) /Apply dynamic prioritization } \\
\text { alarms(EVN) } \\
\text { Error mitigation: Presentcomputer check results to } \\
\text { operators (i.e. check whether all parameters/status meet } \\
\text { reactor/turbine trip criteria) } \\
\text { Present a list of critical tasks with task execution status } \\
\text { and associated plant parameters to operation support staff }\end{array}$ \\
\hline $\begin{array}{l}\text { b. } \\
\text { Diagnose SG } \\
\text { tube rapture }\end{array}$ & $\begin{array}{l}\text { CFM1: PIF- C3, } \\
\text { HSI2,3,5, FS8, } \\
\text { SF0, Inf0 } \\
\text { CFM4: PIF- } \\
\text { C31, HSI2,3,5, } \\
\text { FS2, FS8, SF0, } \\
\text { Inf0 }\end{array}$ & $\begin{array}{l}\text { WO/ } \\
\text { DS: } \\
2.6 \\
\text { W/ } \\
\text { DS: } \\
3.2\end{array}$ & $\begin{array}{l}\text { WO/ } \\
\text { DS: } \\
61 \% \\
\text { W/ } \\
\text { DS: } \\
52 \%\end{array}$ & $\begin{array}{l}\text { Error previsions: Present intuitive cue for EOP-3 entry } \\
\text { condition (e.g., individual alarm of "main steam line } \\
\text { radiation alarm" on Large Display Panel)(HSI) } \\
\text { Error mitigation: Present computer check results to } \\
\text { operators } \\
\text { Present a list of critical tasks with task execution status } \\
\text { and associated plant parameters to operation support staff }\end{array}$ \\
\hline
\end{tabular}

Legend) W/DS(with design solutions), WO/DS(without design solutions).

It is noted that numerical value in the SA and WL column in the table represent hypothetical values because the purpose aims to verify implementation practices.

\subsection{Step 4}

In this phase, human performance of each operation crew is measured in simulator training program. Critical tasks identified in Step 2 are included in training scenarios. If human failures or finding are identified during scenarios, context describing plant and work situation are tracked. Those contexts are fed back to Step 2 and PIFs are adjusted and design countermeasures are reexamined.

\section{Result}

In this application, the HRA/HFE integrated process had specified further detail for PIF attributes which had not been obtained in HRA and showed further investigations to treat how operators induced their human errors through their cognitive task process in their work environment. This application showed effectiveness of the process in order to provide design countermeasures for preventing potential human errors occurrence based on the extensive PIFs and their error forcing context in HRA.

\section{Conclusions}

This paper studied how to integrate HRA and HFE process by employing the latest HRA qualitative 
process, then demonstrate the integration process with the sample event showing the difference in the current HRA quantitative analysis results.

The results show HRA qualitative analysis results (i.e., CFMs/PIFs) are directly communicated with HFE process (i.e., task analysis) not just by screening with risk importance thresholds, then CFMs/PIFs, which are currently used for HEPs calculations, are used for HSI development and procedure development as a design requirement in HFE process in order to minimize potential human error occurrences.

Conflict of Interest

The authors confirm that there is no conflict of interest to declare for this publication.

Acknowledgments

This work is supported by Mitsubishi Heavy Industries, Ltd., and Tottori University in Japan.

\section{References}

Boring, R.L., \& Bye, A. (2008, September). Bridging human factors and human reliability analysis. In Human Factors and Ergonomics Society 52 ${ }^{\text {nd }}$ Annual Meeting, 52(11), 733-737. Los Angeles. USA. DOI:10.1177/154193120805201108.

Hart, S.G., \& Staveland, L.E. (1988). Development of NASA-TLX (Task Load Index): results of empirical and theoretical research. Advances in Psychology, 52, pp 139-183, North Holland Press. USA.

Kirwan, B., \& Ainisworth, L.K. (1992). A guide to task analysis. Taylor \& Francis, London, UK. Chapter 9 (pp.267-362). https://doi.org/10.1201/b16826.

U.S. Nuclear Regulatory Commission. (2007). Guidance for the review of changes to human actions, NUREG-1764, Rev.1, U.S. NRC, Washington D.C., USA.

U.S. Nuclear Regulatory Commission. (2012). Building a psychological foundation for human reliability analysis, NUREG-2114, U.S. NRC, Washington D.C., USA.

U.S. Nuclear Regulatory Commission. (2012). Human Factors Engieering Program Review Model. NUREG-0711, Rev.3, November 2012., U.S. NRC, Washington D.C., USA.

U.S. Nuclear Regulatory Commission. (2020). Integrated human event analysis system for event and condition assessment (IDHEAS-ECA), RIL-2020-02, U.S. NRC, Washington D.C., USA. 